\title{
Comparative analyses of foregut and hindgut bacterial communities in hoatzins and cows
}

\author{
Filipa Godoy-Vitorino ${ }^{1,2,7}$, Katherine C Goldfarbb ${ }^{3,7}$, Ulas Karaoz ${ }^{3}$, Sara Leal ${ }^{4}$, \\ Maria A Garcia-Amado ${ }^{5}$, Philip Hugenholtz ${ }^{2,6}$, Susannah G Tringe ${ }^{2}$, Eoin L Brodie ${ }^{3}$ \\ and Maria Gloria Dominguez-Bello ${ }^{1}$ \\ ${ }^{1}$ Department of Biology, University of Puerto Rico, Rio Piedras, San Juan, Puerto Rico; ${ }^{2}$ Microbial Ecology \\ Program, DOE Joint Genome Institute, Walnut Creek, CA, USA; ${ }^{3}$ Ecology Department, Earth Sciences \\ Division, Lawrence Berkeley National Laboratory, Berkeley, CA, USA; ${ }^{4}$ Centro de Investigaciones Ecológicas \\ de Guayana (CIEG), Universidad Nacional Experimental de Guayana (UNEG), Estado Bolivar, Venezuela; \\ ${ }^{5}$ Instituto Venezolano de Investigaciones Cientificas (IVIC), Caracas, Venezuela and ${ }^{6}$ Australian Centre for \\ Ecogenomics, School of Chemistry and Molecular Biosciences and Institute for Molecular Bioscience,
} The University of Queensland, St Lucia, Australia

\begin{abstract}
Foregut fermentation occurs in mammalian ruminants and in one bird, the South American folivorous hoatzin. This bird has an enlarged crop with a function analogous to the rumen, where foregut microbes degrade the otherwise indigestible plant matter, providing energy to the host from foregut fermentation, in addition to the fermentation that occurs in their hindguts (cecum/colon). As foregut fermentation represents an evolutionary convergence between hoatzins and ruminants, our aim was to compare the community structure of foregut and hindgut bacterial communities in the cow and hoatzin to evaluate the influences of host phylogeny and organ function in shaping the gut microbiome. The approach used was to hybridize amplified bacterial ribosomal RNA genes onto a high-density microarray (PhyloChip). The results show that the microbial communities cluster primarily by functional environment (foreguts cluster separately from hindguts) and then by host. Bacterial community diversity was higher in the cow than in the hoatzin. Overall, compared with hindguts, foreguts have higher proportions of Bacteroidetes and Spirochaetes, and lower proportions of Firmicutes and Proteobacteria. The main host differences in gut bacterial composition include a higher representation of Spirochaetes, Synergistes and Verrucomicrobia in the cow. Despite the significant differences in host phylogeny, body size, physiology and diet, the function seems to shape the microbial communities involved in fermentation. Regardless of the independent origin of foregut fermentation in birds and mammals, organ function has led to convergence of the microbial community structure in phylogenetically distant hosts.

The ISME Journal (2012) 6, 531-541; doi:10.1038/ismej.2011.131; published online 22 September 2011

Subject Category: microbial population and community ecology

Keywords: microbiota; foregut; hindgut; hoatzin; cow; PhyloChip
\end{abstract}

\section{Introduction}

The co-evolution of animals and microbes led to the development of mutualistic relationships between hosts and their microbial colonizers, accounting for an expansion of the host's metabolic traits (Stevens and Hume, 1995; Hooper and Gordon, 2001; Backhed et al., 2005). Thus, animals across the phylogenetic tree have, to varying degrees, a portion of the gastrointestinal tract adapted to accommodate fermenting microbes, which assist in the digestive

Correspondence: MG Dominguez-Bello, Department of Biology, University of Puerto Rico, Rio Piedras Campus, PO BOX 23360,

San Juan 00931-3360, Puerto Rico.

E-mail: maria.dominguez1@upr.edu

${ }^{7}$ These authors contributed equally to this work.

Received 11 April 2011; revised 9 August 2011; accepted 15

August 2011; published online 22 September 2011 process. In these enlarged gut regions, dense communities of microorganisms form a close ecological unit with the host, having a vital role in the nutrition, physiology and immunology of the host animal (Langer, 1991). In the gut microbial chambers, microbial extracellular enzymes catalyze the hydrolysis of the refractory dietary plant fiber that otherwise could not be degraded by the animal's enzymes (Russell and Rychlik, 2001).

Animals can be classified into foregut or hindgut fermenters, based on the characteristics of their digestive fermentation sites. By definition, a foregut fermenter has a pre-gastric fermentation chamber whereas a hindgut fermenter has enlarged fermentation compartments in the cecum and/or colon (Stevens and Hume, 1998). The cow rumen is the most thoroughly studied foregut ecosystem. The microbial processes therein involve fermentation of 
sugars, starches, cellulose, hemicellulose and pectins into $\mathrm{CO}_{2}$, methane, $\mathrm{H}_{2}$ and short chain volatile fatty acids (Stevens and Hume, 1998). These short chain volatile fatty acid, including acetate, propionate and butyrate, are the host's major energy source and are directly absorbed into the portal blood and transported to the liver for gluconeogenesis before reaching the general circulation. In foregut fermenters, bacterial cells are degraded later in the acidic stomach, where gastric lysozyme acts as a digestive enzyme (Ruiz et al., 1994) and bacterial biomass is the animal's main source of protein (Stevens and Hume, 1995). The rumen harbors all three domains of life: bacteria (mostly Firmicutes and the PrevotellaBacteroides (Tajima et al., 2001a; Eckburg et al., 2005)); archaea (such as Methanobrevibacter and Thermoplasma (Tajima et al., 2001b)); and eukarya, both protozoa (ciliates and flagellates; (Orpin, 1976; Vogels et al., 1980) and fungi (anaerobic phycomycetes (Mackie, 1997)).

Additional fermentation occurs in the large bowel and cecum of the cow, where the fermentable substrates are limited to the slower-digesting polymers such as lignin and crystalline starches escaping foregut digestion and absorption, as well as some secreted mucins (Van Soest, 1994). Hindgut symbiotic bacteria continue the fermentation and also provide important vitamins for the host, such as vitamin $\mathrm{K}$, thiamine and riboflavin (Burkholder and McVeigh, 1942; Nath and Meghal, 1961). Fermenting recalcitrant substrates requires longer retention times. Cattle digestive turnover rates can vary from 1-3 days depending on the diet (Hartnell and Satter, 1979).

Foregut fermentation is not a unique trait of cattle, it is also found in other Artiodactyla (sheep, deer, giraffes and antelopes) as well as in marsupials, sloths and Colobus monkeys (Mackie, 2002). Strict herbivory is rare amongst birds, presumably due to mass tradeoffs associated with flight; however, a few birds such as the South American hoatzin (Opisthocomus hoazin) feed on terrestrial plants. The hoatzin is a folivorous bird, unique in possessing an enlarged crop with microbial fermentation (Grajal et al., 1989). This bird is a browser that feeds primarily on tender young plant leaves, from $\sim 17$ plant species (dominated by the plant genus Coccoloba). It has developed special anatomical features to allocate the voluminous crop, including a modification of the sternum and pectoral girdle to accommodate the filled crop. Given its low nutrient density nutritional source, the hoatzin additionally developed a callosity on the breast skin where the heavy crop is rested on a branch (Grajal, 1995). The gastrointestinal system of the hoatzin is therefore composed of a large muscular crop where active foregut fermentation occurs (Grajal et al., 1989) divided in two chambers and a posterior esophagus where additional fermentation occurs. A long small intestine allows autoenzymatic digestion in this species. Caeca are short but the presence of short chain volatile fatty acids and a low $\mathrm{pH}$ indicate the presence of fermentation (Grajal, 1995). The enlarged hoatzin crop has a diverse microbiota of bacteria, archaea, fungi and ciliates that has been studied in detail through molecular ecological methods (Godoy-Vitorino et al., 2008). The crop bacteria include a high abundance of Bacteroidetes, Firmicutes, Proteobacteria and Actinobacteria, and several other less abundant phyla. Phylogenetic novelty in the crop ecosystem is very high at the genus and species level compared with most other gut ecosystems (Godoy-Vitorino et al., 2008, 2010).

Given that foregut fermentation is a case of evolutionary convergence between hoatzins and ruminants, we sought to compare the community structure of foregut and hindgut bacterial communities to test how host phylogeny and organ function contribute to shaping gut communities.

\section{Materials and methods}

Animals

Four adult hoatzins were captured in the Orinoco river (San Ignacio stream, Las Galderas, Bolívar state, Venezuela) during the rainy season, under permit number 3187, obtained from the Venezuelan Ministry of Environment. Captures were done in early morning by shooting the animals that were roosting in the top branches of trees. We immediately dissected the crop and ceca in situ. Crop contents with pieces of leaves and stems still intact, denoted that birds had recently eaten. Whole crops and ceca with their contents were sealed (tied with a cord) in their posterior and anterior ends and immediately frozen in liquid nitrogen and transported to the University of Puerto Rico in dry ice where DNA was extracted. Sampling protocol was approved by the UPR-IACUC.

Four pasture-fed cows were sampled at the 'Macelo La Muda' Slaughterhouse in Guaynabo, Puerto Rico, under authorization of a USDA Veterinarian. We obtained the gut contents immediately postmortem and sub-sampled $\sim 50 \mathrm{ml}$ into a centrifuge tube of each rumen and colon contents from each animal, which were frozen until DNA extraction.

\section{DNA extraction}

DNA was extracted from contents of four hoatzin crops and ceca and four cow rumens and colons. Samples were designated as follows: CR1-4 (cow rumen), CC1-4 (cow colon), HC1-4 (hoatzin crop) and Hce1-4 (hoatzin ceca). The foreguts presented solid particles including intact leaves and stems in the case of the hoatzin crop and intact forage in the cow rumen. The hindguts had both a thick liquid and sand-like texture. DNA was extracted from $\sim 200 \mathrm{mg}$ of bulk contents from each foregut (crop and rumen) and hindgut (ceca and colon). DNA from 
the biological material of the hoatzin and cow organs, was extracted using the QIAamp DNA stool mini kit (Qiagen, Valencia, CA, USA). We modified the first step of the extraction protocol by adding $0.5 \mathrm{~g}$ of sterile $0.1 \mathrm{~mm}$-diameter zirconium beads (Biospec Products, Bartlesville, OK, USA), and $1 \mathrm{ml}$ buffer ASL (Qiagen) to each $200 \mathrm{mg}$ of crop contents, and homogenizing (5000 r.p.m. for $2 \mathrm{~min}$ at room temperature) in a bead beater (Biospec Products). DNA samples were stored frozen $\left(-20^{\circ} \mathrm{C}\right)$ until use.

Amplification of $16 S$ rRNA genes

PCR was performed using universal bacterial primers 27F (5'-AGRGTTTGATCMTGGCTCAG-3') and 1492R (5'-GGTTACCTTGTTACGACTT-3'; Lane, 1991). The $50 \mu \mathrm{l}$ PCR mixtures contained $25 \mu \mathrm{l}$ of PCR Master Mix (Promega, Madison, WI, USA), $50 \mathrm{ng}$ of DNA template and $10 \mathrm{pmol}$ of each primer. For each of the 16 samples, 8 replicate PCR amplifications were performed, with a range of annealing temperatures from $48-58{ }^{\circ} \mathrm{C}$ (gradient PCR), with an initial denaturation at $95{ }^{\circ} \mathrm{C}(3 \mathrm{~min})$, followed by 25 cycles of denaturation at $95^{\circ} \mathrm{C}(30 \mathrm{~s})$, annealing (30 s), extension at $72{ }^{\circ} \mathrm{C}(2 \mathrm{~min})$ and a final extension at $72{ }^{\circ} \mathrm{C}(10 \mathrm{~min})$. The multiple PCR products for each sample were verified for correct product formation by electrophoresis on a $2 \%$ agarose gel. The PCR products were pooled and purified with a PCR purification kit (Qiagen), and used for both clone library construction and hybridization onto the $16 \mathrm{~S}$ rRNA gene microarray.

PhyloChip G2 microarray processing and data analyses Microarray analysis was performed using the G2 PhyloChip, an Affymetrix-platform microarray (West Sacramento, CA, USA) with 506944 probes arranged in 712 rows and columns, representing $\sim 8400$ bacterial taxa, with at least 1 order of magnitude of sensitivity higher than that of a clone library with hundreds of clones (DeSantis et al., 2007).

Each one of the 506944 array 'spots' is around 1 million DNA oligos-all the same sequence. Probes are grouped into different sets that distinguish among 8741 distinct taxa, representing 121 bacterial and archaeal orders, 455 families and 842 subfamilies (Brodie et al., 2006; DeSantis et al., 2007). The probes were designed where possible to only identify one sequence, but some spots contain probes that cover a few or many sequences $(0-3 \%$ sequence divergence). These sequences are then contained in a species to genus-level operational taxonomic unit (OTU) and a representative organism/sequence is chosen for that OTU (Brodie et al., 2006). The G2 PhyloChip can perform quantitative comparisons as was demonstrated previously by the strong linear relationship between PhyloChip intensities and quantities of bacterial 16S rRNA gene signatures applied to PhyloChips (Brodie et al., 2007).
The detailed protocols have been reported previously (Brodie et al., 2006). Briefly, after the DNA was amplified as previously explained, amplicons were fragmented with DNAse (Invitrogen, Carlsbad, CA, USA), biotin labeled, denatured and hybridized to the microarray at $48{ }^{\circ} \mathrm{C}$ overnight ( $>16 \mathrm{~h}$ ). The arrays were subsequently washed and stained. Reagents, conditions and equipments involved are detailed elsewhere (Masuda and Church, 2002). Scanning of the arrays was performed using a GeneArray Scanner (Affymetrix) and probe intensities were treated as reported previously (Brodie et al., 2006). Positive probe pairs met two criteria as follows: (1) fluorescence of the perfectly matched probe was at least 1.3 times greater than the intensity of the control (mismatch probe) and (2) the value of the difference between perfectly matched probe and mismatch probe intensities was at least 130 times greater than the squared noise value. The value of the positive fraction was calculated for each probe set as the number of positive probe pairs divided by the total number of probe pairs in a probe set. A positive fraction $\geqslant 0.90$ was used to denote the presence/absence of an OTU/taxon.

The normalized intensities were then scaled by the average overall array intensity to account for variation in PCR amplicon quantification, and those values were log transformed to normalize variance at different intensities. The normalized, log-transformed intensity values were used for further analysis (Ivanov et al., 2009).

\section{Data analysis}

Overall, richness was calculated as the sum of taxa present in each sample. Richness estimates for a particular gut section are reported as the mean of four replicates (with s.d.).

All statistical analyses were based on taxa present in at least three out of four samples for either gut section in each organism. Statistical analyses were carried out in the R software environment (http:// www.R-project.org). A distance matrix was calculated from the normalized log-transformed intensity values using the Bray-Curtis distance metric within the function 'vegdist' in the R package 'vegan'. The distance matrix was represented as a nonmetric multidimensional scaling plot using the function 'metaMDS' and variance partitioning was calculated with the function 'adonis'. Statistical significance of gut section or animal groupings was determined by analysis of similaries, relative group variance homogeneity was verified with a multivariate analogue of Levene's test (function 'betadisper') using the same Bray-Curtis distance matrix. Rank abundance curves were plotted using only normalized logtransformed intensities for taxa that were considered present in a given replicate. Inverse Simpson's index was calculated with the function 'diversity'. 
Phylum level percent composition was calculated as the percentage of taxa in a given phylum for a given core community relative to the total number of taxa in that given core community. All core/unique taxa analysis was based on the presence/absence threshold, and does not reflect taxon relative abundance.

To determine taxa with significantly enriched abundance in one gut section relative to the other for a given host animal, we applied a one-way analysis of variance and difference of means $(P \leqslant 0.05$ deemed significant). Analogous comparisons were made for taxa significantly enriched in a particular host animal for a given gut section. Heatmaps were drawn using Pearson's correlation as the similarity metric and average linkage clustering in ' $R$ ' using the package heatmap (Eisen et al., 1998).

We also performed unweighted UniFrac clustering (with jackinfing) using FastUniFrac (Hamady et al., 2010). We compared bacterial communities of different foregut and hindgut fermenters (cloning sequences) as well as the G2 chip detected OTUs from our study. We included sequences from the Zebra, Horse, Wild Ass, Banteng, Indian and Black Rhinoceros, Capybara, Gazelle, Giraffe, Okapi, Kangaroo, Springbok, Takin and Sheep all from (Ley et al., 2008). We also included sequences from other studies that satisfied the inclusion criteria of sequence length $>500 \mathrm{bp}$, and data sets containing $>50$ sequences from each particular host species, these included: sequences from the dairy cow rumen (Chin EC, Lim WJ, Kim $\mathrm{H}$ and Yun HD unpublished data available in the GenBank database); sequences from cow manure (Nakai Y and Yamamoto $N$, unpublished data available in the GenBank database), sequences from the chicken cecum (Massias B, Urdaci MC unpublished data available in the GenBank database), sequences from Turkey cecum (Scupham et al., 2008) and Hoatzin clone sequences (Godoy-Vitorino et al., 2008). The hoatzin and cow G2Chip OTUs, correspond to the representative sequences of the OTUs detected by the PhyloChip through hybridization with foregut and hindgut DNA from the wild Venezuelan Hoatzins and pasturing cows (this study).

\section{Results}

\section{Bacterial community structure}

Amplification of 16S rRNA genes from all samples and hybridization to the PhyloChip revealed unique communities for each host and organ type. Nonmetric multidimensional scaling analysis of the results shows that the gut communities cluster both by functional environment and by host (Figure 1a). Analysis of similarity showed that foregut and hindgut communities (axis 1) were significantly different $(P<0.001)$, as were hoatzin and cow communities (axis 2; $P<0.002$ ), although the latter is not as clear as axis 1 due to the higher dispersion of the samples of hoatzin cecum, suggesting that organ function may be an even more important driver of community composition than host species. Hoatzin samples, in particular the ceca, showed higher dispersion in the nonmetric multidimensional scaling plot analysis than cow organs, as confirmed by the significantly higher inter-individual variability in the hoatzin $(P=0.002$; Figure $1 b)$. Variance between foreguts or hindguts was similar (Figure 1c).

To determine common bacterial community composition, core gut taxa were defined as taxa present in at least three out of four replicates for samples in a particular gut division. For example, the core foregut community contained taxa present in at least three out of four replicates from the hoatzin crop and the cow rumen (Supplementary Figure 1A). Core animal communities were similarly defined between animal hosts across gut sections (Supplementary Figure 1B). Further, a 'super core' community was defined as common taxa between complementary core communities (that is, overlap between core foregut and core hindgut or core hoatzin and core cow communities). Gut unique bacteria were defined as taxa not present in the core communities, for example the cow rumen specific community was comprised of taxa present in three out of four replicates of cow rumen but absent from the core cow community (Supplementary Figures 1A, B).

Overall, the cow digestive organs (rumen and colon) were richer (higher number of (species to genus-level) OTUs than the hoatzin's $(P=0.0035$; Figure 2a; Supplementary Figure 2A). The cow rumen and hindgut were also more diverse based on Simpson's indices (Figure 2b). The hoatzin crop was particularly even, compared with all other organs (Figure 2c). The hindgut rank-abundance curves clearly showed that the cow colon is richer and more even when compared with the hoatzin ceca (Supplementary Figure 2B).

There were 33 bacterial phyla indicated to be present across all the organs and hosts (Supplementary Figure 3). The core foregut microbiota (OTUs common to all hoatzin and cow foregut samples; Supplementary Figure 1A) contained 464 OTUs in at least three out of four replicate animals, whereas the core hindgut microbiota contained 365 OTUs. The communities were fairly similar at the phylum level, with the foreguts having a slightly greater number of OTUs from Bacteroidetes $13 \%$ vs $9 \%$ in hindguts) and Actinobacteria (5\% vs $2 \%$ in hindguts) and a lower richness of Firmicutes (33\% lower than the $38 \%$ in the hindguts) and Proteobacteria (22\% vs $24 \%$ in hindguts; Supplementary Figure 3).

The digestive organ was the primary determinant of microbial community structure within each animal gut. There were 160 OTUs that were unique to foreguts and not present in hindguts, including OTUs belonging to the Bacteroidetes, Cyanobacteria, Lentisphaerae, Planctomycetes, Spirochaetes and 
a

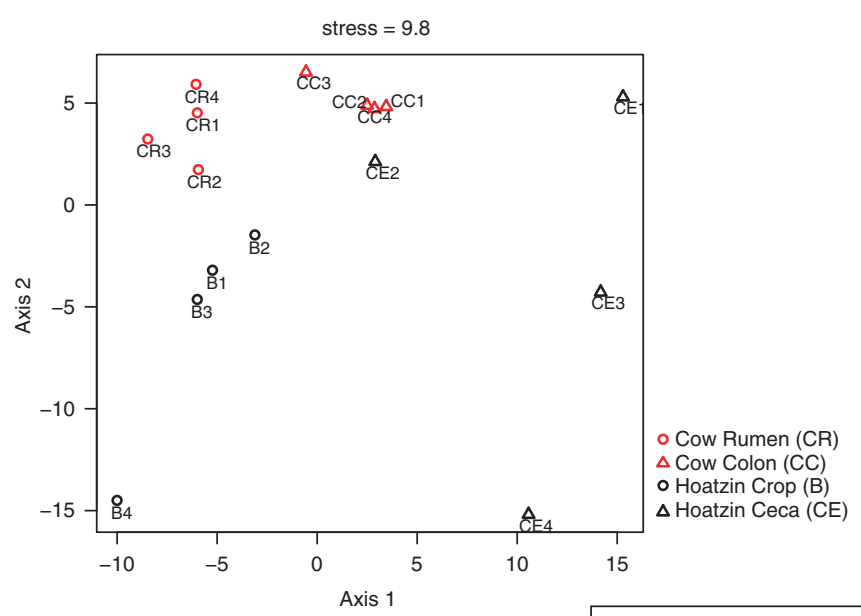

b

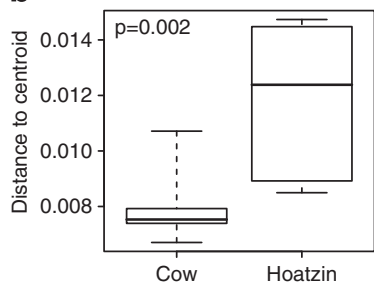

C

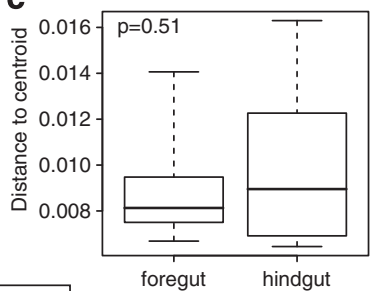

Anosim statistic: Difference of means

$R$ (gut site) $=0.395, \quad p<0.001$

$R($ host animal) $=0.28, p=0.002$

Figure 1 Bacterial community structure. (a) Nonmetric multidimensional scaling of community structure in the foregut and hindgut samples of hoatzins and cows; circles represent the foreguts and triangles represent the hindguts. Dotted line helps to visually define axis 1. (b) Analyses of dispersion for the communities within each animal host and (c) analyses of dispersion for each organ site.
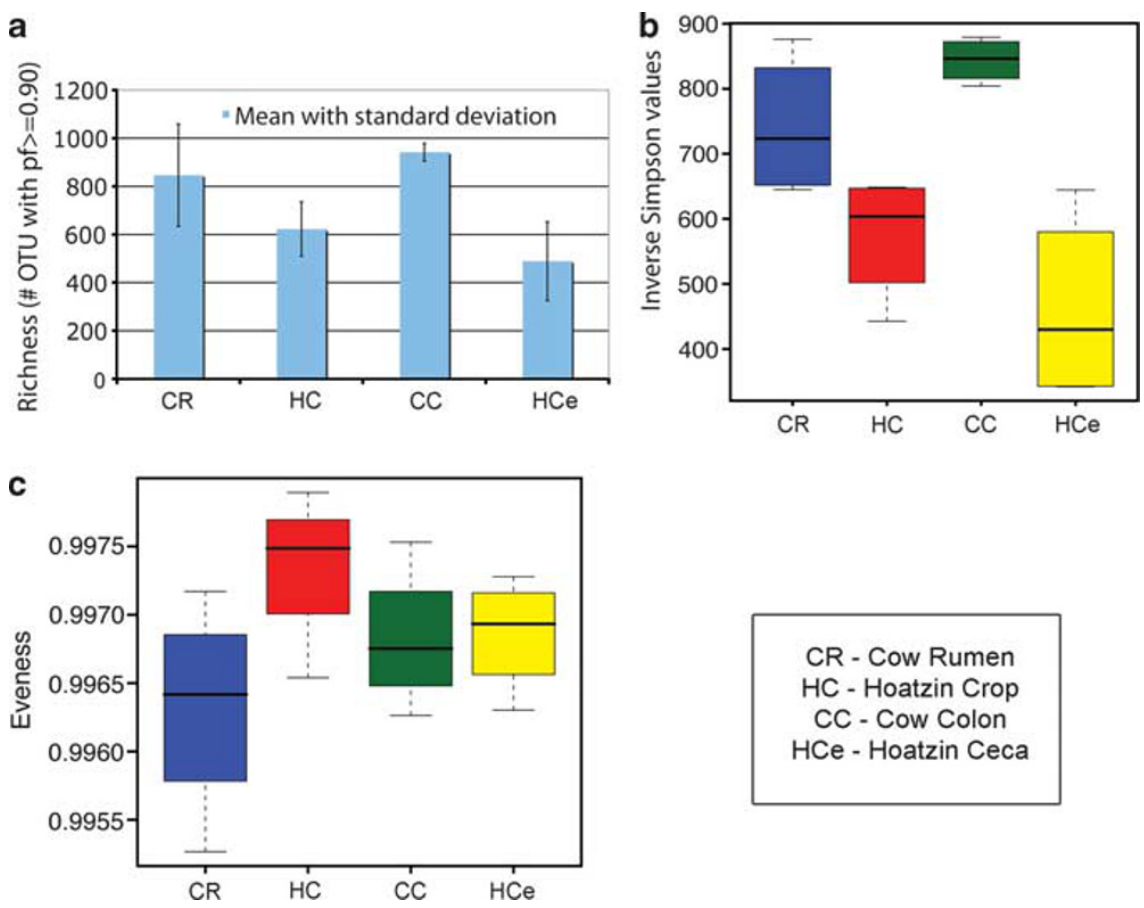

Figure 2 Richness and diversity analyses for each host organ. (a) Mean richness, (b) inverse Simpson's values and (c) evenness.

candidate phylum TM7. For the cow, there were 370 significantly different OTUs between the rumen and colon, particularly among the Spirochaetes and Bacteroidetes (Flavobacteria and Sphingobacteria); those enriched in the colon include Proteobacteria, Actinobacteria, Chloroflexi and Firmicutes (class Symbiobacteria) (Figure 3, Supplementary Table S1). For the hoatzin, there were 285 OTUs with significantly different relative abundances in the crop and ceca of the hoatzin. Similar to cows, the hoatzin crop also had an enriched complement of Bacteroidetes, and fewer Proteobacteria and Firmicutes OTUs than the ceca (Figure 3, Supplementary Table S2). Overall, foreguts were enriched in populations of Bacteriodetes, Acidobacteria and Spirochaetes, and contained fewer OTUs belonging to the Proteobacteria and Firmicutes than the hindguts (Figure 3). 


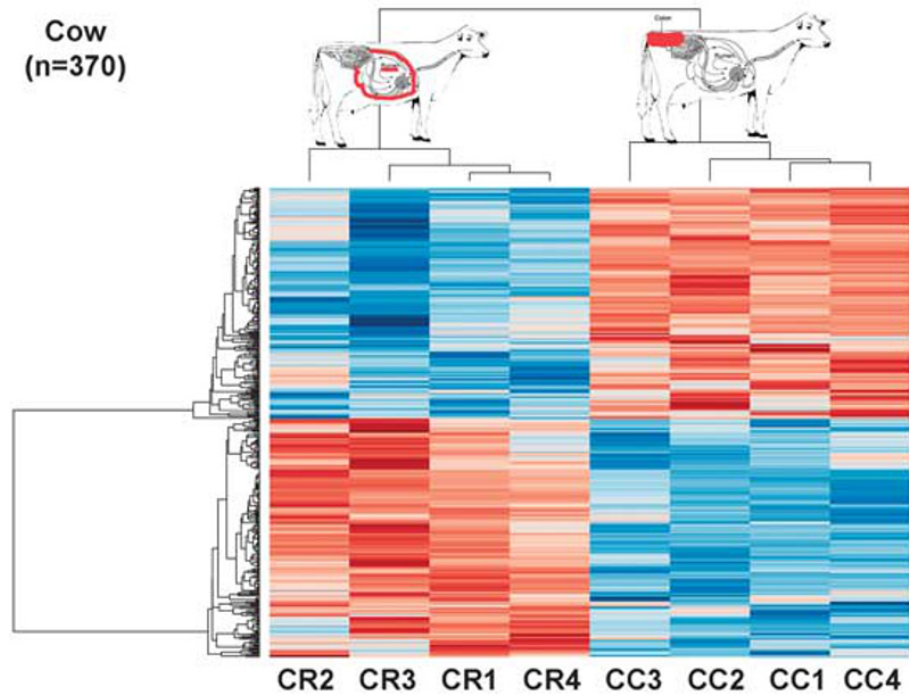

a Up in Cow Colon $(n=188)$

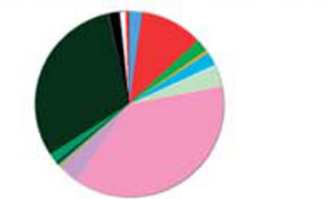

b Up in Cow Rumen $(n=182)$

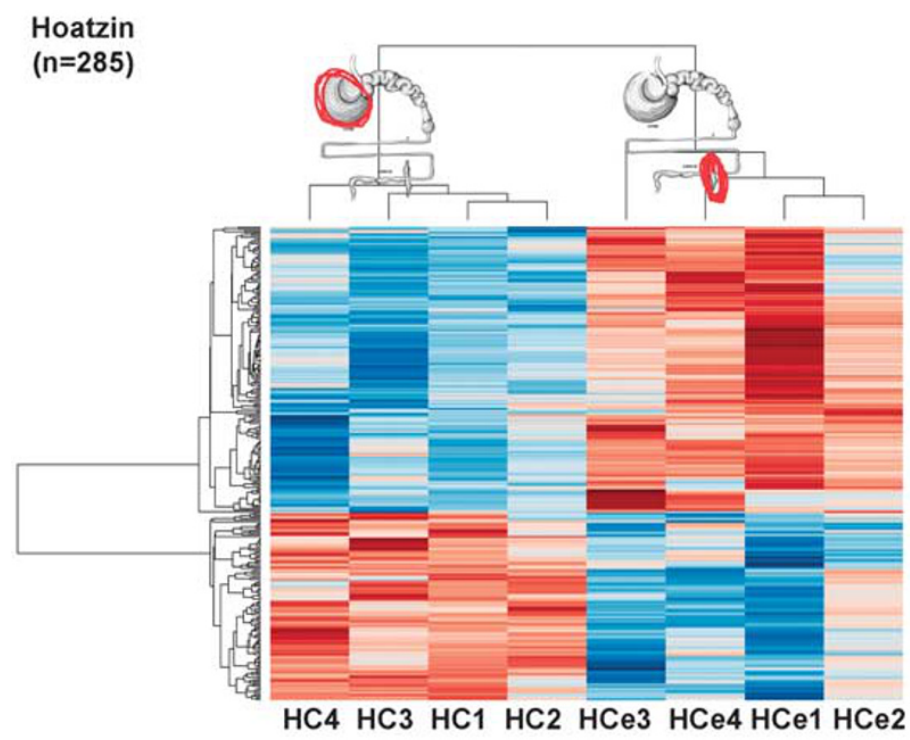

C Up in Hoatzin Ceca $(n=172)$
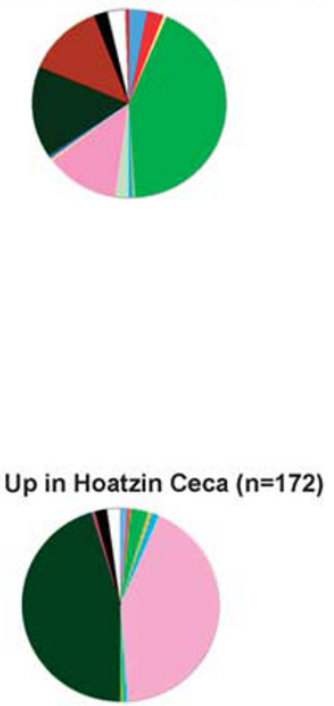

d Up in Hoatzin Crop $(n=113)$

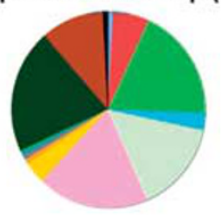

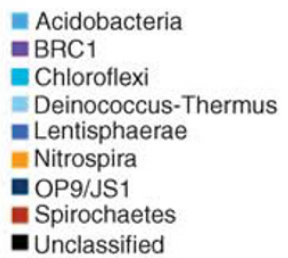

\begin{tabular}{|c|}
\hline $\begin{array}{l}\text { Actinobacteria } \\
\text { Caldithrix } \\
\text { Coprothermobacteria } \\
=\text { Firmicutes } \\
\text { marine group A }\end{array}$ \\
\hline
\end{tabular}

Aquificae

- Chlamydiae

- Cyanobacteria

= Gemmatimonadetes

= Natronoanaerobium

ㅂ. $\mathrm{OP} 3$

- Proteobacteria

- Thermodesulfobacteria

WS3

Figure 3 Heatmaps with bidirectional clustering of bacterial OTUs and specific organ communities, showing the relationship between the foregut and hindgut within host. The heatmap of the top panel displays the relationship between the eight cow samples (four rumen and four colon) and the 370 significantly different taxa. The pie charts on the right depict: (a) the phyla of the 188 OTUs that are significantly more abundant in the cow colon, whereas in (b) are the phyla of the 182 OTUs that are significantly more abundant in the cow rumen. Detailed taxonomic description of each OTU can be found in Supplementary Table S1. The heatmap of the bottom panel depicts the relationship between the eight hoatzin samples (four crop and four ceca) and the 285 OTUs that changed significantly between each gut site. The pie charts on the right depict: (c) the phyla of the 172 OTUs that are significantly more abundant in the hoatzin ceca, whereas in (d) are the Phyla of the 113 OTUs that are significantly more abundant in the hoatzin crop. Detailed taxonomic description of each OTU can be found in Supplementary Table S2.

A total of 304 OTUs were common to the core foregut and core hindgut (Supplementary Figure 3). The OTUs that were exclusive to the core hindgut $(n=61)$ included members of the bacterial phyla
Gemmatimonadetes, Firmicutes, WS3 and SPAM (Supplementary Figure 4). The core microbial richness (OTUs shared between the foregut and hindgut) was higher for the cow (625 OTUs) than for the 


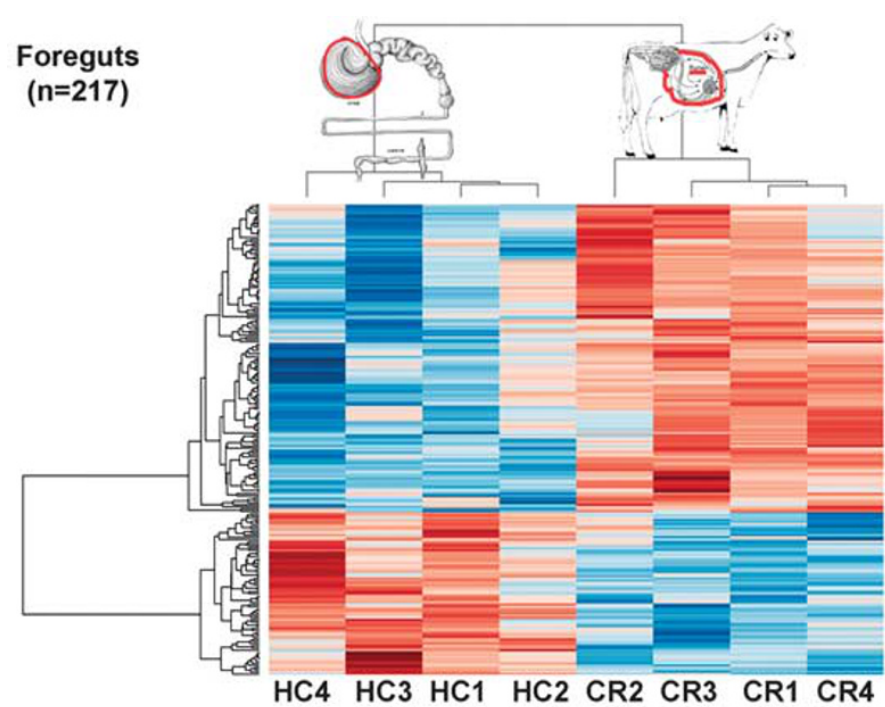

a Up in Cow Rumen ( $n=142)$
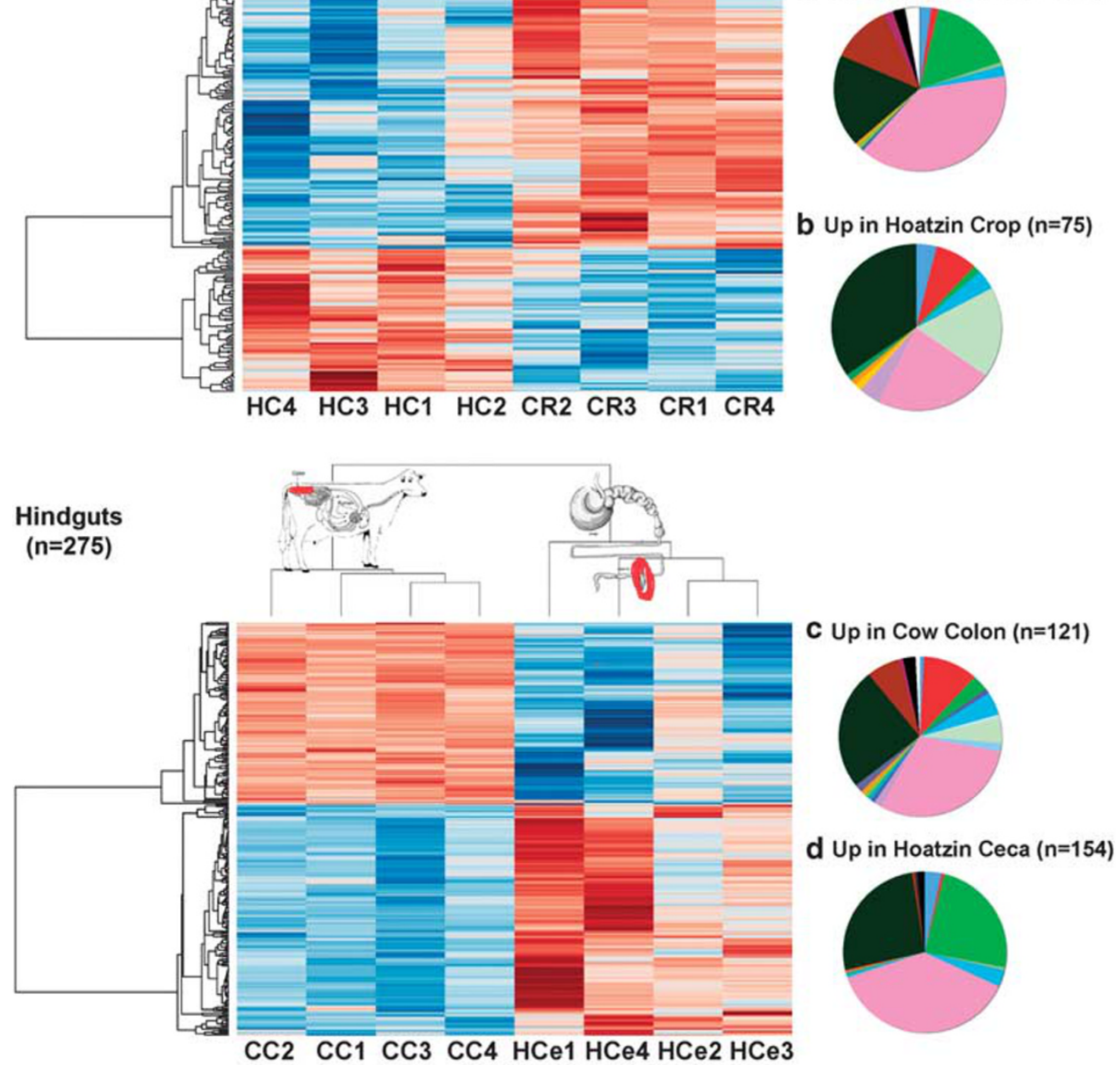

- Acidobacteria
- BRC1
- Chloroflexi
= Deinococcus-Thermus
n Lentisphaerae
= Nitrospira
- OP9/JS1
- Spirochaetes
- Unclassified

= Actinobacteria
= Caldithrix
Coprothermobacteria
= Firmicutes
= marine group A
= OP10
= Planctomycetes
= Synergistes
$\square$ Verrucomicrobia

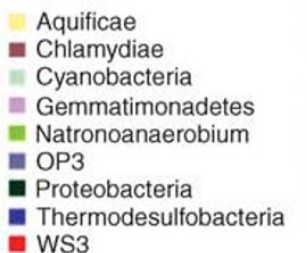

- Bacteroidetes

Enlorobi

Deferribacteres

LD1PA group

NC10

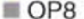

- SPAM

- $\mathrm{DM} 7$

Figure 4 Heatmaps with bidirectional clustering of bacterial OTUs and specific organ communities, showing the inter-host relationship between the foreguts and hindguts. The heatmap of the top panel displays the relationship between eight the 217 significantly different taxa of all foreguts (four crops and four rumen). The pie charts on the right depict: (a) the phyla of the 142 OTUs that are significantly more abundant in the cow rumen and in (b) the phyla of the 75 OTUs that are significantly more abundant in the hoatzin crop. Detailed taxonomic description of each OTU can be found in Supplementary Table S3. The heatmap of the bottom depicts the relationship between the 275 OTUs that changed significantly between the hindguts (four colons and four ceca). The pie charts on the right show: (c) the phyla of the 121 OTUs that are significantly more abundant in the cow colon and in (d) the phyla of the 154 OTUs that are significantly more abundant in the hoatzin ceca. Detailed taxonomic description of each OTU can be found in Supplementary Table S4.

hoatzin (311 OTUs; Supplementary Figure 5). Although profiles were similar at the phylum level, there were only seven OTUs unique to the hoatzin, whereas the cow had 321. The OTUs unique to the cow had a higher proportion of Proteobacteria,
Actinobacteria and Spirochaetes (Supplementary Figure 5).

A total of 217 OTUs were significantly different between the cow rumen and the hoatzin crop (Figure 4, Supplementary Table S3). The crop had 
a greater proportion of unclassified OTUs and OTUs belonging to the Gemmatimonadetes, Planctomycetes and candidate phylum NC10. The hoatzin had a higher representation of certain Proteobacterial groups such as the orders Azospirillales and Bradyrhizobiales (Alphaproteobacteria), and Alteromonadales and Oceanospirillales (Gammaproteobacteria). The cow rumen had a higher abundance of Chlorobi, Lentisphaerae, Spirochaetes, Synergistes and Verrucomicrobia (Figure 4, Supplementary Table S3). A higher proportion of Firmicutes in the cow included OTUs belonging to the families Clostridiaceae and Peptostreptococcaceae, whereas a higher proportion of Bacteroidetes was represented in the orders Flavobacteriales and Sphingobacteriales (Supplementary Table S3).

For the hindguts, there were 275 OTUs that differed significantly between the cow colon and hoatzin ceca, with the cow colon having higher proportion of Spirochaetes, Actinobacteria, Cyanobacteria, Synergistes, Nitrospirae, DeinococcusThermus or Verrucomicrobia. The cow colon exhibited a predominance of Actinobacteria (Cellulomonadaceae, Micromonosporaceae or Kineosporiaceae and Rubrobacterales) that were absent in the hoatzin ceca. The very few OTUs with higher relative abundance in the hoatzin ceca than in the cow colon belong to the bacterial phyla Chlorobi, TM7, Bacteroidetes (including those in the order Sphingobacterales) and also some Firmicutes (Figure 4, Supplementary Table S4).

Because similarities in community structure were found in both gut sites in the bird and cow, with existing 'core' communities between both hosts, we performed a UniFrac community analyses using other animals with foregut and hindgut fermentation including mammals and birds. UniFrac is based on the premise that related communities share an evolutionary history that can be estimated as the fraction of shared branch length in a common phylogenetic tree. We used 8643 16S rRNA sequences including the representative OTUs detected by the PhyloChip in our study (Figure 5a). The clustering demonstrated that indeed the hoatzin microbial communities are mostly similar to those of the foregut fermenters and divergent from those of other birds such as chicken and turkey, both of which cluster with hindgut fermenter animals. With the limitations of detecting only known bacterial taxa with the PhyloChip and of having cloned a limited number of clones, the analysis that excluded the PhyloChip OTUs (Figure 5b), shows that the hoatzin is closest to mammalian foregut fermenters. Phylum-level similarities between the bird and cow are also presented (Supplementary Figure 6).

\section{Discussion}

Our study provides evidence that organ function is a stronger determinant of microbial community structure than is host phylogeny. There is a great similarity between the foregut and hindgut microbiotas of both hosts (core microbiotas) regardless of the expected weight of host physiology, body size and diet. The foreguts exhibit a higher relative abundance in Bacteroidetes, whereas the hindguts show a higher relative abundance in Proteobacteria and Firmicutes. In fact, the broad UniFrac comparison showed that the hoatzin microbiota is more similar to that of foregut fermenter mammals than to organs from other birds (chickens and turkey), indicating a strong selective pressure of herbivory.

Differences in organ microbial community structure include the location relative to absorption sites, volumes and retention times. Foreguts are preabsorption organs, and in foregut fermenters they are, in relation to hindguts, more voluminous and have characteristic longer retention times and more heterogeneous digesta (Stevens and Hume, 1995). Foreguts also tend to fill and empty, whereas volume fluctuations are less marked in the hindgut. The foregut contents are mostly solid with entire plant parts from the diet, whereas the contents of the hindgut are more homogeneous with already predigested plant material (Stevens and Hume, 1995). Indeed the hoatzin's crop contents were highly solid with pieces of leaves or entire small leaves intact, whereas the contents of the ceca were liquid with a sand-like texture. The same observations were made with the cow rumen where ruminal contents were bulky and coarse whereas the colon contents were heavily liquid and macerated with unidentifiable plant material. The microbiota does change depending on whether it is associated with solid or liquid fractions (Rodriguez et al., 2000) and with the quality of fermentable substrate (more recalcitrant in the hindgut (Van Soest, 1994)).

Previous results have shown differences in composition of the microbiota along the length of the gut, in the mouse (Wang et al., 2010), chicken (Rehman et al., 2007) and humans (Costello et al., 2009). Both host diet and phylogeny influence fecal bacterial diversity, as shown by Ley et al. (2008) who based on the composition of the feces, showed that herbivores generally clustered into two groups corresponding to foregut and hindgut fermenters. Exceptions were folivore primates such as the Colobus monkey and the François Langur, with microbial lineages similar to those in omnivores. Our study shows that a bird folivorous browser such as the hoatzin possesses a core foregut and hindgut bacterial lineages in common with the cow rumen and colon, respectively, thus showing that organ function is indeed an important driver of the bacterial ecosystem composition, despite host phylogenetic distances.

Differences between foreguts and hindguts include the gradient decline in water content (Hecker and Grovum, 1971), $\mathrm{pH}$ (buffering effect of the bile and bicarbonate (Mackie and Wilkins, 1988)), 
a

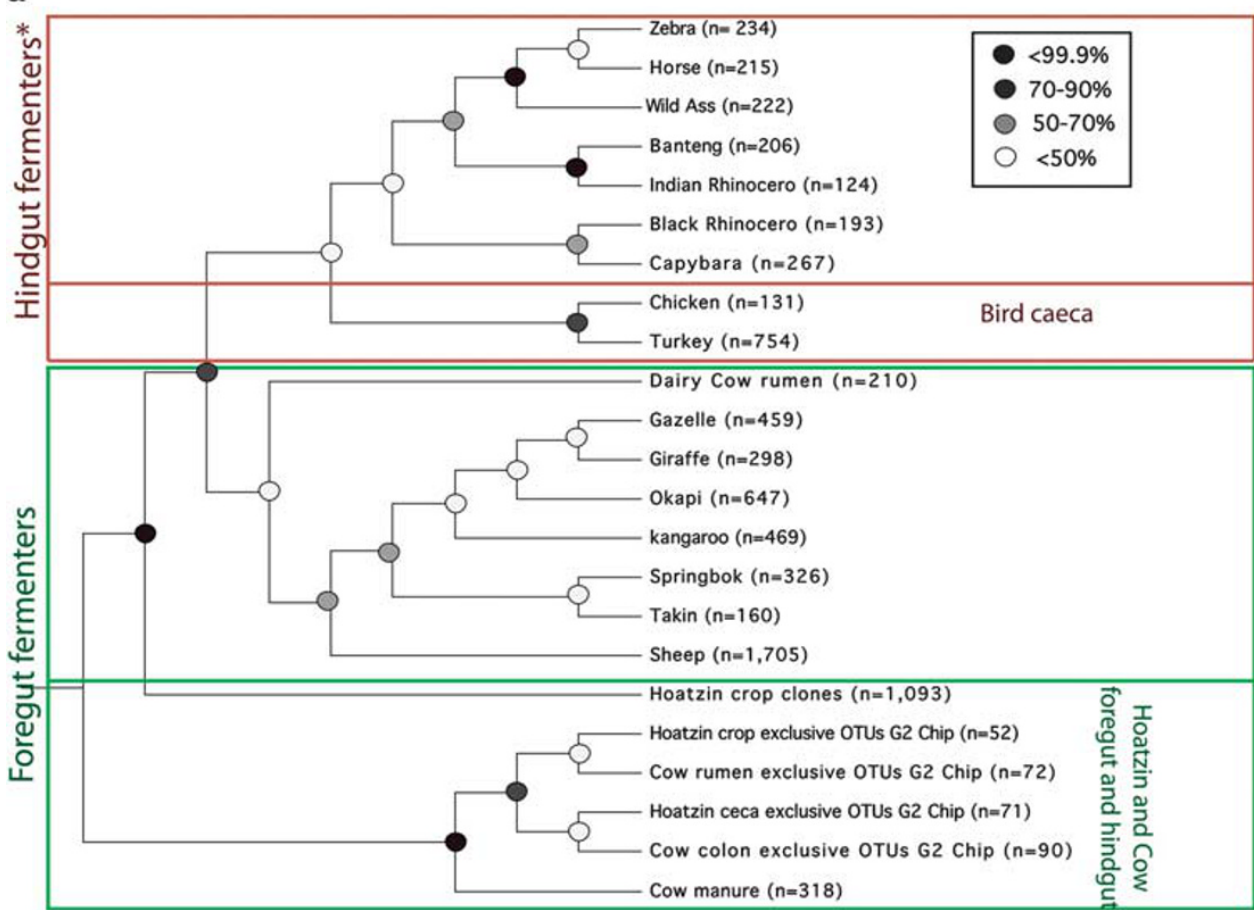

b

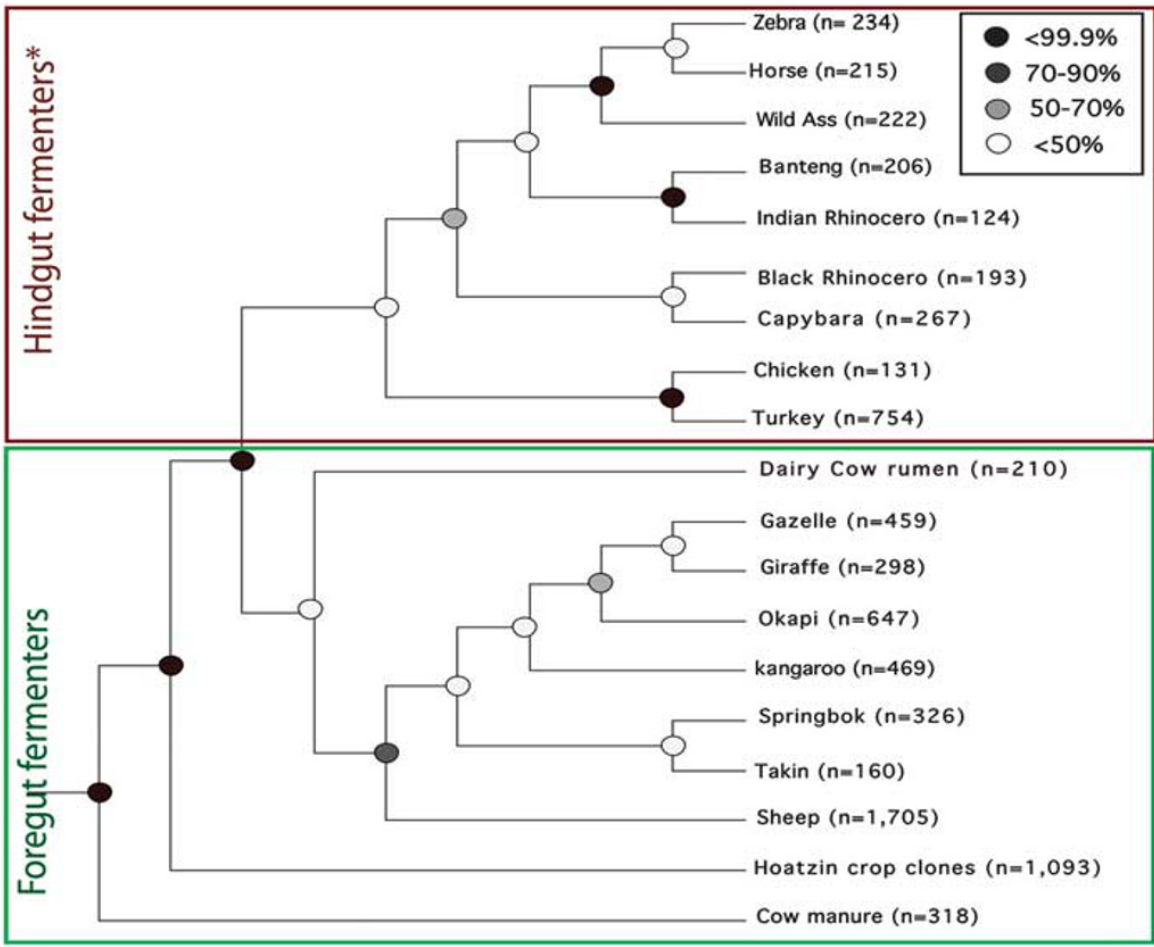

Figure 5 UniFrac community analyses comparing the Hoatzin and cow to other foregut and hindgut fermenter animals. (a) Unweighted clustering of the bacterial communities of different foregut and hindgut fermenters including cloned sequences and the G2 chip detected OTUs from our study. (b) Unweighted clustering of the different foregut and hindgut bacterial communities excluding the G2 chip representative OTUs.

particle size and VFA concentration fluctuations (Sato and Shiogama, 2009).

The cecum has fermentation characteristics similar to the colon but differs in the retention of selected feed fractions (Van Soest, 1994), and in that it empties in pulses behaving like a batch culture (Van Soest, 1994). The passage rate is difficult to calculate especially in a bird, and this may explain the fact that the hoatzin ceca are highly dispersed (high variance). 
These physico-chemical differences between both gut regions lead to significant differences in the microbiota of each host. The higher representation of Bacteriodetes and Spirochaetes in the foregut may be related to higher cellulolytic activity, whereas hindgut dominance by Proteobacteria and Firmicutes might be related to higher proteolytic activity (Appleby, 1955; Mackie and Wilkins, 1988).

That the cow rumen was richer than the hoatzin crop is likely linked to habitat size, in direct relation with richness (Chesson, 2000), as predicted by the theory of island biogeography (MacArthur and Wilson, 1967). Longer retention times in cows compared with the hoatzin $(72 \mathrm{~h}$ vs $44 \mathrm{~h}$, respectively; (Hartnell and Satter, 1979; Grajal, 1991)) are possible due to the larger fermentation chambers that sustain more extensive fiber digestion. A higher relative abundance of Spirochaetes, Verrucomicrobia and Lentisphaerae in the cow rumen is consistent with high cellobiose degradation (Zoetendal et al., 2003; Warnecke et al., 2007). Indeed, the rumen has been shown to be more efficient in degrading cellulose than the hoatzin crop (Jones et al., 2000).

Diet is surely a major determinant in shaping digestive communities (Tajima et al., 2001a), and there are major differences between the cow and hoatzin diet. The hoatzin is a browser, consuming green leaves, bark and green stems from young plants, low in fiber and high in nitrogenous compounds, whereas the cow is a grazer that feeds on grasses (monocots). There are major chemical differences between monocot and dicot cell walls. Legumes and most dicots contain smaller proportions of hemicellulose in their cell walls than monocots (Van Soest, 1994) whereas these have extensive interconnecting networks of phenylpropanoids (Iiyama et al., 1990), which may help to explain why the cow rumen has a higher proportion of Bacteroidetes than the crop, member of this phylum breakdown these lignin components (Akin, 1988).

This comparative work shows that the similarity in the microbial composition between these evolutionarily distant hosts is a case of evolutionary convergence. Despite the considerable phylogenetic divergence between the hosts and dietary differences there are strong similarities in the foregut and hindgut communities of hoatzins and cows. We conclude that host characteristics (that is, phylogeny, diet, size and weight) are less important than the functional niche of the organ for differentiating bacterial community composition.

\section{Acknowledgements}

This work was supported by Grants from NSF IOS 0716911, NSF DEB-DDIG 0709840 and NSF CREST/ HRD0206200. Part of this work was performed at the Lawrence Berkeley National Laboratory under the auspices of the University of California-contract number DE-AC02-05CH11231. We gratefully acknowledge fieldwork support from José González-Fernandez, Juan González-Fernandez and Antonio González-Fernandez from Hato Mataclara (Cojedes river) where the birds were captured. We also thank Mr Jorge Morales (owner of Macelo-La Muda Slaughterhouse) and Dr Enid Avilés (USDA Veterinarian) the authorization of the sampling procedures of cow rumen and colon contents.

\section{References}

Akin DE. (1988). Biological structure of lignocellulose and its degradation in the rumen. Anim Feed Sci Tech 21: 295-310.

Appleby JC. (1955). The isolation and classification of proteolytic bacteria from the rumen of the sheep. J Gen Microbiol 12: 526-533.

Backhed F, Ley RE, Sonnenburg JL, Peterson DA, Gordon JI. (2005). Host-bacterial mutualism in the human intestine. Science 307: 1915-1920.

Brodie EL, Desantis TZ, Joyner DC, Baek SM, Larsen JT, Andersen GL et al. (2006). Application of a highdensity oligonucleotide microarray approach to study bacterial population dynamics during uranium reduction and reoxidation. Appl Environ Microbiol 72: 6288-6298.

Brodie EL, DeSantis TZ, Parker JP, Zubietta IX, Piceno YM, Andersen GL. (2007). Urban aerosols harbor diverse and dynamic bacterial populations. Proc Natl Acad Sci USA 104: 299-304.

Burkholder PR, McVeigh I. (1942). Synthesis of vitamins by intestinal bacteria. Proc Natl Acad Sci USA 28: 285-289.

Chesson P. (2000). Mechanisms of maintenance of species diversity. Annu Rev Ecol Syst 31: 343-366.

Costello EK, Lauber CL, Hamady M, Fierer N, Gordon JI, Knight R. (2009). Bacterial Community Variation in Human Body Habitats Across Space and Time. Science 326: 1694-1697.

DeSantis TZ, Brodie EL, Moberg JP, Zubieta IX, Piceno YM, Andersen GL. (2007). High-density universal 16S rRNA microarray analysis reveals broader diversity than typical clone library when sampling the environment. Microbial Ecol 53: 371-383.

Eckburg PB, Bik EM, Bernstein CN, Purdom E, Dethlefsen L, Sargent M et al. (2005). Diversity of the human intestinal microbial flora. Science 308: 1635-1638.

Eisen MB, Spellman PT, Brown PO, Botstein D. (1998). Cluster analysis and display of genome-wide expression patterns. Proc Natl Acad Sci USA 95: 14863-14868.

Godoy-Vitorino F, Goldfarb K, Brodie E, Garcia-Amado MA, Michelangeli F, Dominguez-Bello MG. (2010). Developmental microbial ecology of the crop of the folivorous hoatzin. ISME J 4: 611-620.

Godoy-Vitorino F, Ley RE, Gao Z, Pei Z, Ortiz-Zuazaga H, Pericchi LR et al. (2008). Bacterial community in the crop of the hoatzin, a neotropical folivorous flying bird. Appl Environ Microbiol 74: 5905-5912.

Grajal A. (1991). Digestive efficiency of the hoatzin (Opisthocomus hoatzin), a folivorous bird with foregut fermentation. PhD dissertation. University of Florida: Gainesville, FL, 111pp. 
Grajal A. (1995). Structure and function of the digestive tract of the hoatzin (Opisthocomus hoazin): a folivorous bird with foregut fermentation. The Auk 112: 20-28.

Grajal A, Strahl SD, Parra R, Dominguez MG, Neher A. (1989). Foregut fermentation in the Hoatzin, a Neotropical Leaf-Eating Bird. Science 127: 1131-1134.

Hamady M, Lozupone C, Knight R. (2010). Fast UniFrac: facilitating high-throughput phylogenetic analyses of microbial communities including analysis of pyrosequencing and PhyloChip data. ISME J 4: 17-27.

Hartnell GF, Satter LD. (1979). Determination of rumen fill, retention time and ruminal turnover rates of ingesta at different stages of lactation in dairy cows. J Anim Sci 48: 381-392.

Hecker JF, Grovum WL. (1971). Absorption of water and electrolytes from the large intestine of sheep. Aust $J$ Biol Sci 24: 365-369.

Hooper LV, Gordon JI. (2001). Commensal host-bacterial relationships in the gut. Science 292: 1115-1118.

Iiyama K, Lam TBT, Stone BA. (1990). Phenolic acid bridges between polysaccharides and lignin. Phytochemistry 29: 733-773.

Ivanov II, Atarashi K, Manel N, Brodie EL, Shima T, Karaoz $\mathrm{U}$ et al. (2009). Induction of intestinal Th17 cells by segmented filamentous bacteria. Cell 139: 485-498.

Jones RJ, Amado MA, Dominguez-Bello MG. (2000). Comparison of the digestive ability of crop fluid from the folivorous Hoatzin (Opisthocomus hoazin) and cow rumen fluid with seven tropical forages. Anim Feed Sci Tech 87: 287-296.

Lane DJ. (1991). 16S/23S rRNA Sequencing. Wiley: London, pp 115-175.

Langer P. (1991). Evolution of the digestive tract in mammals. Verh Dtsch Zool Ges 84: 169-193.

Ley RE, Lozupone CA, Hamady M, Knight R, Gordon JI. (2008). Worlds within worlds: evolution of the vertebrate gut microbiota. Nat Rev Microbiol 6: 776-788.

MacArthur RH, Wilson EO. (1967). The Theory of Island Biogeography. Princeton: New Jersey, USA.

Mackie RI. (1997). Gut environment and evolution of mutualistic fermentative digestion. In: ba MRaW (ed). Gastrointestinal Microbiology. Chapman and Hall: New York, pp 13-35.

Mackie RI. (2002). Mutualistic fermentative digestion in the gastrointestinal tract: diversity and evolution. Integr Comp Biol 42: 319-326.

Mackie RI, Wilkins CA. (1988). Enumeration of anaerobic bacterial microflora of the equine gastrointestinal tract. Appl Environ Microbiol 54: 2155-2160.

Masuda N, Church GM. (2002). Escherichia coli gene expression responsive to levels of the response regulator EvgA. J Bacteriol 184: 6225-6234.

Nath MC, Meghal SK. (1961). Effect of carbohydrates on the intestinal synthesis of thiamine in rats. Biochem J 81: 220-224.

Orpin CG. (1976). Studies on the Rumen Flagellate Sphaeromonas communis. J Gen Microbiol 94: 270-280.
Rehman HU, Vahjen W, Awad WA, Zentek J. (2007). Indigenous bacteria and bacterial metabolic products in the gastrointestinal tract of broiler chickens. Arch Anim Nutr 61: 319-335.

Rodriguez CA, Gonzalez J, Alvir MR, Repetto JL, Centeno C, Lamrani F. (2000). Composition of bacteria harvested from the liquid and solid fractions of the rumen of sheep as influenced by feed intake. Br J Nutr 84: 369-376.

Ruiz MC, Dominguez-Bello MG, Michelangeli F. (1994). Gastric lysozyme as a digestive enzyme in the hoatzin (Opisthocomus hoazin), a ruminant-like folivorous bird. Experientia 50: 499-501.

Russell JB, Rychlik JL. (2001). Factors that alter rumen microbial ecology. Science 292: 119-1222.

Sato H, Shiogama Y. (2009). Acetone and isopropanol in ruminal fluid and feces of lactating dairy cows. $J$ Vet Med Sci 72: 297-300.

Scupham J, Patton TG, Bent E, Bayles DO. (2008). Comparison of the cecal microbiota of domestic and wild turkeys. Microb Ecol 56: 322-331.

Stevens CE, Hume ID. (1995). Comparative Physiology of The Vertebrate Digestive System. Cambrige University Press: Cambridge, UK.

Stevens CE, Hume ID. (1998). Contributions of microbes in vertebrate gastrointestinal tract to production and conservation of nutrients. Physiol Rev 78: 393-427.

Tajima K, Aminov RI, Nagamine T, Matsui $H$, Nakamura M, Benno Y. (2001a). Diet-dependent shifts in the bacterial population of the rumen revealed with real-time PCR. Appl Environ Microbiol 67: $2766-2774$.

Tajima $K$, Nagamine $T$, Matsui $H$, Nakamura $M$, Aminov RI. (2001b). Phylogenetic analysis of archaeal 16S rRNA libraries from the rumen suggests the existence of a novel group of archaea not associated with known methanogens. FEMS Microbiol Lett 200: 67-72.

Van Soest PJ. (1994). Nutritional Ecology of the Ruminant, 2nd edn Comstock: Ithaca, xii, 476pp.

Vogels GD, Hoppe WF, Stumm CK. (1980). Association of methanogenic bacteria with rumen ciliates. Appl Environ Microbiol 40: 608-612.

Warnecke F, Luginbuhl P, Ivanova N, Ghassemian M, Richardson TH, Stege JT et al. (2007). Metagenomic and functional analysis of hindgut microbiota of a wood-feeding higher termite. Nature 450: 560-565.

Wang Y, Devkota S, Musch MW, Jabri B, Nagler C, Antonopoulos DA et al. (2010). Regional mucosaassociated microbiota determine physiological expression of TLR2 and TLR4 in murine colon. PLOS ONE 5: e13607.

Zoetendal EG, Plugge CM, Akkermans AD, de Vos WM. (2003). Victivallis vadensis gen. nov., sp. nov., a sugarfermenting anaerobe from human faeces. Int $J$ Syst Evol Microbiol 53: 211-215.

Supplementary Information accompanies the paper on The ISME Journal website (http://www.nature.com/ismej) 